\title{
La mise en jeu du corps dans l'activité de téléphonie.
}

The Place of the Body in Call Center Activity

\section{Anne-France Kogan}

\section{(2) OpenEdition}

Journals

Édition électronique

URL : http://journals.openedition.org/edc/1059

DOI : 10.4000/edc. 1059

ISSN : 2101-0366

Éditeur

Université Lille-3

Édition imprimée

Date de publication : 1 décembre 2009

Pagination : 171-186

ISSN : $1270-6841$

Référence électronique

Anne-France Kogan, « La mise en jeu du corps dans l'activité de téléphonie. », Études de communication [En ligne], 33 | 2009, mis en ligne le 01 décembre 2011, consulté le 30 avril 2019. URL : http://journals.openedition.org/edc/1059; DOI : 10.4000/edc.1059

Ce document a été généré automatiquement le 30 avril 2019

(c) Tous droits réservés 


\section{La mise en jeu du corps dans l'activité de téléphonie.}

The Place of the Body in Call Center Activity

Anne-France Kogan

\section{Introduction : activité et corporéité}

1 Nous proposons dans cet article de relire différents travaux sur les centres d'appel en questionnant la mise en jeu du corps dans l'activité de téléphonie. Le choix de ce regard s'appuie sur l'hypothèse qu'il existe un lien entre l'activité d'information et la posture corporelle. L'activité d'information est ici entendue « comme étant ce qui se joue autour et avec l'information, c'est à dire celle qui implique les acteurs dans l'alimentation de systèmes collectifs» (Guyot, 2002, p13). Cet élargissement est important car les missions assurées par les téléopérateurs dans ces centres d'appels oscillent du recueil d'informations simples au conseil personnalisé pour la résolution de problèmes techniques par exemple. Autrement dit, le système d'information, s'il préside largement à la construction de la réponse apportée par le téléopérateur lors d'un appel qu'il soit émis ou reçu, est également en partie alimenté par l'activité des téléopérateurs, qui, par exemple, participent à enrichir et transformer la base de données par des informations sur les appelants et le contenu des appels.

2 Les travaux récents de la sociologie du travail mobilisent également la notion d'activité qui puise dans les traditions phénoménologique et pragmatistei. La phénoménologie affirme le corps comme sujet de perception et le pragmatisme ancre toute perception dans l'agir. Cette perspective permet alors d'aborder «le travail comme une activité, entre corps et technique, et permet « de saisir sa dynamique créatrice et les ressorts de l'engagement au travail, entre ennui et créativité, assurance et vulnérabilité, focalisation et circulation, aisance et perplexité " (Bidet, 2007b, p215). Cette approche est particulièrement féconde pour analyser le travail immatériel, ou plus précisément un «travail de plus en plus équipé en dispositifs médiatisés, automatisés et plus généralement en abstraction ». (Bidet, 2007a, p19). 
3 Apparu au milieu des années quatre vingt dix, les centres d'appels téléphoniques sont fondés sur une alliance du téléphone et de l'informatique. La spécificité du travail, fortement équipé en Tic, nous amène à interroger la manière dont ces équipements contraignent le travail et pèsent sur les salariés. En effet, pour réaliser cette activité, l'opérateur porte un combiné ou un casque qui est couplé au dispositif de saisie (écran, clavier, souris) lui permettant d'identifier le client et l'arrivée de l'appel (sa fiche apparaît sur l'écran) ou de suivre le "script téléphonique " à l'écran dans le cas des appels sortants. L'importance de cet équipement technique et la contrainte d'attachement physique au dispositif de télécommunication et d'information réduisent les possibilités de mouvement du corps.

4 C'est donc à la lumière de cette notion d'activité que nous proposons de relire les recherches menées dans les centres d'appel. Il s'agit dès lors de considérer, d'une part, ce qui se déploie autour de cette communication téléphonique postée et cadrée par le système d'information, et, d'autre part, comment y est abordée la corporéité.

\section{Une modernité désenchantée}

5 La première lecture de ces travaux apparait comme un manifeste dénonçant la pénibilité des nouveaux métiers associés à cette activité. La force du propos est renforcée par le décalage entre ces mauvaises conditions de travail et les espoirs nourris par les possibilités qu'offrent les Tic pour produire un service à distance. En effet, les Tic, en repoussant les frontières spatio-temporelles des organisations, ouvraient la porte à de nouvelles promesses, et apparaissaient aux yeux de beaucoup comme le vecteur majeur de transformations économiques et sociales. Plus particulièrement dans le secteur des services où « des pans entiers, qui ne dépendent pas d'un contact physique directe entre l'offreur et le client potentiel, peuvent être déplacés à des milliers de kilomètres de leur marché de destination, sans que cet éloignement ne constitue une entrave à l'exécution des tâches, sans même souvent que le consommateur final ait conscience de cet éloignement »(Pichault, Zune, 2000, p1).

6 Au-delà de la rationalisation excessive qui est souvent dénoncée, nous chercherons à saisir ce qui caractérise cette activité et la place donnée à la corporéité. En effet, derrière ces promesses, une autre réalité est apparue :

«devenus synonymes d'une forme moderne de dégradation des conditions de travail et d'emploi des salariés, les centres d'appel font la une des journaux autour des conflits sociaux qu'ils suscitent, de la faible protection juridique de leurs salariés ou de la nature taylorisée des emplois créés. Les centres d'appel seraient des usines modernes, les employés des plates-formes téléphoniques les nouveaux OS du tertiaire ». (Buscatto, 2002, p100).

7 Une deuxième lecture permet de nuancer le propos car il existe une hétérogénéité des conditions de travail qui n'est pas sans lien avec l'hétérogénéité des centres d'appels euxmêmes. Une première distinction se fait sur le fait que les communications téléphoniques sont, soit données par les téléopérateurs (appels sortants), soit reçues au centre d'appel par les télé-conseillers à l'initiative des clients (appels entrants). Au-delà de la simple distinction technique,

« ces deux types d'appels ont une importance non négligeable sur l'organisation du travail et sur les comportements attendus du personnel. Les appels sortants peuvent en effet être programmés de manière précise en fonction de la durée moyenne des appels et de la plage horaire visée et font appel à des techniques 
d'entretien orientées vers la vente ou la persuasion. A l'inverse, les appels entrants sont caractérisés par l'imprévisibilité des intentions des consommateurs et de leurs demandes, les comportements attendus des téléconseillers étant dans ce cas davantage de l'ordre de l'aide, du support, de la prise en charge ou alors du conseil» (Pichault, Zune , 2000, p3).

Un travail plus récent apporte un bémol au constat de mauvaises conditions de travail systématiques dans les centres d'appel, et va jusqu'à dénoncer «le misérabilisme qui prévaut parfois et qui tend à occulter l'hétérogénéité des situations rencontrées en termes de conditions de travail ». (Sarfati, 2008, p31).

Il existe d'autres distinctions qui influent sur les conditions de travail comme la nature des contrats qui lient les donneurs d'ordres aux centres d'appel ou la complexité des savoir-faire qu'exige la réponse aux appels entrants. Cette dernière dimension, à savoir le niveau de technicité des réponses et des contenus des échanges informationnels, distingue en grande partie le degré de pression du flux des appels et donc les conditions de travail. Autrement dit, plus la compétence est élevée, «plus la pression se réduit, car, d'une part, la qualité du service requiert du temps et, d'autre part, la rareté de la maind'œuvre qualifiée acceptant de travailler dans les CAT $^{\text {ii }}$ conduit à la ménager relativement » (Durand, 2003, p168).

Le secteur dans lequel s'opère ce service intervient également sur la pression du flux et le stress qu'il génère : ainsi, dans le secteur de la téléphonie sociale ${ }^{\text {iii, }}$ la logique productive s'efface derrière celle de l'aide. Les téléopérateurs, qu'ils soient salariés ou bénévoles sont donc beaucoup moins soumis à cette pression.

11 Nous choisirons de distinguer quatre types de centres d'appels. Une première distinction s'opère entre ceux qui réalisent des appels sortants (i) qui recrutent en général avec un faible niveau de qualification (appels commerciaux), et ceux qui réalisent des appels entrants. Parmi eux, on peut distinguer ceux (ii) qui effectuent une assistance technique mobilisant différents niveaux de qualification (assistance technique des opérateurs télécom, informatiques, assurance, etc.), ceux (iii) qui coordonnent une assistance à distance (rapatriement, téléassistance pour personnes âgées), et enfin ceux (iv) qui s'inscrivent dans la téléphonie sociale.

Quelque soit ces distinctions, ces travaux ne s'intéressent pas directement à la corporéité mais nous pouvons néanmoins repérer quelques éléments. Nous présenterons tout d'abord les traits communs au travail des télé-opérateurs pour ensuite caractériser les quatre types d'appels identifiés.

\section{La spécificité de l'activité téléphonique : un corps entravé, un travail sous tension}

Comme nous l'avons déjà évoqué, pour réaliser cette activité, l'opérateur est fortement équipé : entre le casque d'écoute, et l'écran pour suivre le « script téléphonique » et saisir des informations, le téléopérateur se trouve "attaché» au dispositif technique et voit donc ses mouvements corporels réduits. Autrement dit, l'écoute est une activité contraignante en raison de son caractère posté. A partir d'une analyse d'un cas clinique d'une téléopératrice, deux psychologues cliniciens pointent les effets sur le corps :

«celle-ci (la logique productiviste de l'organisation) se manifeste au travers de la machinisation - elle (la salariée interviewée) dit qu'elle se «branche » à minute fixe tous les matins, sent qu'on veut faire d'elle "le clone d'autres clones", est 
" formatée ", " bordée ", de la répétition, des relations amputées avec les collègues, du manque d'autonomie et de la soumission du corps immobilisé en position assise. » (Bouvet, Schneider, 2007, p211) la relation téléphonique, et centrées sur les déterminants organisationnels et managériaux de la santé mentale au travail, fait apparaitre très clairement les tensions vécues par les téléopérateurs.

« L'individu doit incarner les valeurs de l'entreprise, intégrer son discours auprès $\mathrm{du}$ client, exprimer des sentiments non natures (feindre les émotions), voire parfois imaginer des sentiments et un ressenti (se " mettre à la place » du client ou de l'usager) en d'autres termes faire fi de son individualité (émotion, identité, etc.), tout en étant impliqué totalement dans l'échange ». (Clergeau et al., 2009).

15 inhérents à l'activité : il faut respecter les rendements tout en satisfaisant aux exigences $\mathrm{du}$ client, il faut offrir une réponse relativement standardisée tout en respectant la singularité de la demande, sans oublier de considérer l'humeur du client. Car dans la relation de service, l'objet travaillé concerne la vie d'autrui, dès lors, les manières de le réaliser sont beaucoup plus controversées par nature et fondamentalement discutables. Ainsi, contrairement au travail industriel, il est impossible de séparer les opérations d'exécution et le sens de l'action, ce qui impose une responsabilité renouvelée et du coup, la définition des tâches se heurte plus qu'ailleurs aux évaluations conflictuelles. "L'objet" travaillé devenu "sujet" laisse moins encore qu'auparavant les travailleurs en paix. Il multiplie les "problèmes de conscience" » (Clot, 2008, p252). De même, réalisés sur une plate-forme téléphonique, il apparaît que « les scripts, les objectifs quantitatifs ou l'écoute téléphonique ponctuelle sont impuissants à régler des dilemmes qui relèvent des caractéristiques même du produit offert. » (Buscatto, 2002, p111).

sécificité de la relation téléphonique, qui, en anonymisant et en effaçant les corps autorise les débordements agressifs ou émotionnels chez le client, vient renforcer cette tension. Ainsi, pour les techniciens résolvant les problèmes des clients par téléphone, « la difficulté majeure de ce métier réside dans la gestion du rapport au client, pour lequel ils ne sont pas formés : certains correspondants leur raccrochent au nez, d'autres adressent insultes et injures. Les comportements désagréables caractérisent $10 \%$ des appels.» (Durand, 2003, p169).

17 C'est également ce que soulignent les psychologues cliniciens en ajoutant qu'« en outre, ces salariés subissent, au nom de l'entreprise, l'agressivité de clients revendicatifs, et en ressentent d'autant plus fortement les effets que ces organisations ne facilitent pas le retravail par le collectif des chocs émotionnels professionnels. » (Bouvet, Schneider, 2007, p204).

La difficulté et les tensions qui pèsent sur les opérateurs sont d'autant plus fortes qu'elles ne peuvent s'exprimer : pour les psychologues, le travail, au moins en théorie, devrait permettre à une certaine quantité d'agressivité et d'émotion de s'exprimer, et ce, de manière socialement contrôlée, donc recevable. «Mais loin d'offrir un lieu d'expression pour l'agressivité qu'ils engendrent, les centres d'appel la répriment fortement: interdiction de réagir agressivement aux demandes (mêmes agressives) des clients, collectifs professionnels fragilisés et contrôle permanent de toute déviance langagière ou comportementale. » (Bouvet, Schneider, 2007, p206)

Études de communication, 33 | 2009 
Dans nos deux premiers types de centres (appels entrants (i) et appels sortants (ii)), caractérisés par un faible niveau de qualification des salariés, la cause principale de ces conditions de travail dégradées est attribuée à l'hyper rationalisation de l'organisation du travail. Un rythme soutenu, une extrême prescription du travail, un contrôle permanent du nombre d'appels pris par jour ainsi que la durée de ces appels, une double écoute aléatoire, une autonomie réduite et un manque de perspectives d'évolution sont à l'origine de cette pénibilité du travail des téléopérateurs. Que le ministère du Travail se soit saisi de la question, proposant un « label social » pour les centres d'appel, est à cet égard révélateur : « alors que ce secteur présente un fort potentiel de création d'emploi, il se heurte à des difficultés de recrutement et de maintien des salariés en poste » (Bouvet Schneider, 2007, p204). Le travail de chargé de clientèle dans un centre d'appels « est considéré par tous comme un travail répétitif et usant. Une norme implicite, régulièrement énoncée au cours de nos entretiens, définit la durée acceptable de ce travail autour de trois à quatre années. » (Buscatto, 2002, p114)

Ce type d'activité est caractérisé par un turnover élevé (plus de 40\%). Car « la fidélisation des techniciens se heurte à la nature du travail inscrite dans le paradigme du flux tendu » (Durand, 2003, p171). Effectivement, l'organisation par le système d'information est souvent dénoncée comme porteur de cette hyper rationalisation du travail, car porteur d'une logique de productivité à laquelle les plates-formes sont soumises. Celles étudiées par Buscatto soulignent que le « rôle du superviseur est de suivre et d'organiser l'activité à partir du système informatique. (...) Son principal objectif est clairement la productivité, favorisant ainsi l'intensification du rythme de prise d'appels des employés téléphoniques et l'assurance qu'ils ne font pas autre chose» (Buscatto, 2002, p104).

Cette forme autoritaire d'organisation est d'autant plus dénoncée qu'elle se fait au service d'une productivité qui nuit souvent à la qualité de l'interaction avec le client, donc à la qualité du travail effectué et, finalement, au sens du travail individuel (Bouvet, Schneider, 2007).

L'hyper rationalisation de l'activité n'est donc pas seule en cause dans les mauvaises conditions de travail dans les centres d'appel. Un corps entravé, une parole muselée, des dilemmes difficiles à régler viennent également peser sur les difficultés vécues par les téléopérateurs. Nous proposons de regarder maintenant les différentes modalités mises en œuvre dans ces centres d'appel pour faire face aux tensions inhérentes à cette activité de télécommunication pilotée par l'informatique.

\section{Travailler l'agir communicationnel en tension}

L'approche par l'activité, permet de dépasser les distinctions traditionnelles entre travail formel et informel ou travail prescrit et travail réel, et surtout, comme nous l'invite A. Bidet, d'y réintroduire la corporéité pour saisir l'agir créatif. Ainsi, les réponses à ces difficultés, si elles sont souvent présentées comme des formes de résistance, ou de la régulation, sont ici à considérer comme faisant partie de l'activité même.

\section{Se mettre à distance}

Une première modalité pour atténuer ces tensions revient à s'écarter du poste pour désentraver le corps. Quand il n'est pas possible de se débrancher, les salariés cherchent à 
mettre à distance le corps, ainsi «les mesures de protection prises contre l'atteinte physique - ménager sa voix, éloigner le casque de ses oreilles - permettent de tenir «l'agression auditive à distance» (Bouvet, Schneider, 2007, p211). D'autres formes renvoient au fait de s'extraire de l'entrave imposée par le dispositif technique, ainsi, «dans la répartition du travail, un effort est également fait par la hiérarchie pour régulièrement les éloigner de l'activité téléphonique jugée usante : travail administratif, formations, participation à des groupes de travail, parrainage des nouveaux, responsabilité sur une activité parallèle » (Buscatto, 2002, p109).

Cette mise à distance physique n'est autre qu'une des modalités d'une mise à distance psychologique dans le but d'éloigner et atténuer les tensions. C'est une nécessité pour les activités de télé service évoquées ici, car dans la relation de service, « les techniques sont celles de l'usage de soi et des autres plus que de la chose physique. [...] A tous les sens du terme, il (le service) « remet » au travail les transports affectifs de personnes à personnes que chaque sujet entretient dans son histoire propre. Il est "psychologiquement" plus intense et plus chargé. Plus "intérieur » aussi et beaucoup moins délimité par les propriétés physiques et chimiques de la matière » (Clot, 2008, p252).

\section{Construire une dynamique collective}

Dans notre troisième catégorie de centres d'appel (iii), qui correspond à ceux du secteur de l'assurance, de l'assistance-rapatriement ou technique, cette nécessité d'une mise à distance permet non seulement d'atténuer les tensions mais aussi de produire et d'homogénéiser les pratiques. Un article consacré à l'étude du travail dans un centre d'appel dédié aux opérations d'assistance montre que pour atteindre le « haut niveau de fiabilité » qu'exige l'organisation d'un rapatriement au téléphone, il appartient aux opérateurs de « décrocher du poste » pour échanger sur son travail et produire du sens :

«Si les chargés d'assistance se concertent peu sur une même opération d'assistance
qui peut durer plusieurs jours, néanmoins, la proximité géographique des chargés
d'assistance sur les plateaux permet leurs échanges sur ce qu'ils viennent de vivre
(client mécontent, réussite, ...) au cours des pauses ou entre les appels
téléphoniques lorsque ceux-ci sont moins nombreux. C'est à cette occasion que se
constitue une base commune de savoirs partagés ». (Michaux, Rowe, 2004, p70).

Dans chacun des cas, cet échange sur le travail individuel d'écoute, qu'il soit organisé ou non, est avant tout collectif. Ainsi, dans le cas des opérations d'assistance, les auteurs mettent aussi l'accent sur l'importance du travail collectif afin de compléter « de façon essentielle les modes opératoires standardisés pour harmoniser les pratiques individuelles d'analyse des situations de décision, harmonisation fondamentale pour assurer la cohérence et la continuité d'un processus itératif distribué d'assistance rapatriement. » (Michaux, Rowe, 2004, p59).

Dans le secteur de l'assurance, même si elle est analysée comme une forme de résistance à la tension générée par l'activité téléphonique, on observe cette même dynamique collective et vertueuse. L'auteure observe une coopération active à la vie de la plateforme téléphonique.

« Dans les groupes de travail mis en place pour améliorer les scripts, les procédures ou les manipulations informatiques, on observe des participants actifs qui suggèrent de nombreuses idées, qui critiquent les règles jugées inefficaces, qui affirment leurs points de vue » (Buscatto, 2002, p109). 

sens renvoie à l'activité d'information telles que la conçoit B.Guyot, c'est à dire une activité de service où il faut collectivement produire le référentiel des actions pour lui donner du sens (Guyot, 2002).

Dans le cas des services de téléassistance pour personnes âgées, les pompiers mandatés pour recevoir ce type d'appels ont conservé pendant dix-sept ans le référentiel des appels construit pour les secours d'urgence, alors que les appels d'autres natures s'imposaient toujours plus. Le fait de choisir collectivement de maintenir cette inadéquation entre la nature des appels de téléassistance et son référentiel leur servait d'arguments pour montrer le décalage entre leur métier, le prompt secours, et ce que leur imposait la téléassistance pour laquelle ils se revendiquaient incompétents. Autrement dit, refuser la mise en cohérence de l'information sur le travail et le travail exprime le refus d'exercer ce type d'activité (Kogan, 2008).

\section{Re-travailler les émotions : une vrai compétence}

31 Le secteur de la téléphonie sociale (type iv) qui, par définition, autorise "les chocs émotionnels " par téléphone s'avère dès lors très instructif car il a, de fait, dès son origine, dû considérer cette tension psychique dans l'activité des téléopérateurs, car il s'agit, par définition, d'offrir un service à des personnes en détresse.

La téléphonie sociale est aujourd'hui intégrée aux dispositifs de prévention et de soins. Elle constitue ainsi un outil d'accompagnement des politiques de santé publique. Répartie selon des pôles thématiques (sida, suicide, tabac, cancer, drogues, etc.) et populationnels (jeunes, personnes âgées, etc.), ces organisations ont toutes développé une forme d'expertise en matière d'écoute et de conseil. L'objectif étant d'aider l'appelant à faire face, à développer et/ou restaurer son autonomie, éclairer ses choix, favoriser sa réflexion, l'accompagner vers un mieux être. Par définition, il s'agit de jouer sur l'écoute où

« l'émotion n'a pas à être retenue. Déprime, chagrin, violence subie, sexualité, les sujets qui semblaient tabous sont évoqués, détaillés. Tout ou presque peut se raconter, se dévoiler au téléphone. L'écoutant écoute, incite à la parole, évalue. Il est là sans être là. L'écoute n'est pas "béante ". elle soutient, elle canalise. L'appelant dévide son histoire, sans regard, à distance mais si proche de celui qui l'écoute, proche à lui parler dans l'oreille. L'intérêt de ce type d'échange est essentiellement constitué par le jeu entre distance et proximité, qui permet de dévoiler sans être identité tout en vivant un entretien personnalisé » (Cadéac et al. , 2004, p1).

C'est pourquoi, dans ce secteur, l'enjeu est aujourd'hui à la professionnalisation pour faire reconnaître cette compétence à pouvoir jouer entre distance et proximité et montrer que «l'écoutant s'appuie sur une technique du dialogue pour mener l'entretien et s'inspire d'une culture psychologique pour décoder la signification latente des propos de l'appelant, sans toutefois aller jusqu'à l'utilisation courante de la technique psychanalytique » (Chauvière, Frotiée, 1998, p172).

Les plates-formes de téléassistance dédiées à la réception des appels des personnes âgées, qui existent depuis la fin des années soixante-dix, ont vu le nombre d'appels dits « de détresse morale» augmenter, alors que le dispositif était prévu pour envoyer une assistance en cas de chutes. Ainsi, les plates-formes de «prompt secours » initialement 
mandatées se retirent au profit de plate-forme dédiées à cette population qui se développent en mettant en avant les compétences spécifiques pour répondre à cette population (Kogan, 2008). Par exemple, les écoutants apprennent à saisir « la demande de repères » qui n'est jamais formulée directement par les personnes âgées. Cette demande est encore plus forte la nuit et les écoutants, confrontés eux aussi aux «sortilèges» de la nuit, partagent avec les abonnés certains de leurs ressentis. Pour être capable de "gérer leur stress et leurs émotions » et satisfaire la demande, ils reçoivent une formation permanente basée sur «les pathologies du vieillissement, l'écoute en relation d'aide à distance, la gestion des situations d'urgence et de crise, l'analyse des pratiques d'écoute. » (Macé, 2006, p237).

La formation n'est pas la seule modalité mise en œuvre pour réaliser cette mise à distance psychologique. Toujours dans le secteur de la téléphonie sociale, on observe au sein de cette activité des procédures collectives qui permettent de "trouver la bonne distance ", et se rapprocher d'un «idéal de l'écoute » qui consiste à ne pas être directif, à s'abstenir de tout jugement ou de tout conseil, à faire en sorte que s'établisse une sorte de congruence entre le ressenti de l'écoutant et ce que dit l'appelant. Ce travail collectif se fait "à côté » du travail d'écoute, ainsi sur une plate-forme d'aide téléphonique aux personnes suicidaires,

« l'ensemble des écoutes passées est relu et réinterprété à travers les récits oraux et publics de certains appels difficiles, et les prises à l'émotion que ceux-ci suscitent. Le réglage de la distance affective à la souffrance d'autrui devient un accomplissement collectif, organisé autour de l'évocation répétée des appels marquants. Le travail sur soi est équipé et distribué : il s'appuie sur des collectifs (... ), des règles (la charte, ...), des techniques d'écoute, des dispositif de codage et d'évaluation des appels, des appuis pour l'action... » (Licoppe, 2008, p425).

Associer le travail d'écoute individuelle à une pratique collective qui vient transformer l'organisation ne peut se faire que si les téléopérateurs bénéficient d'une certaine autonomie, ce qui est le cas, quand ceux-ci possèdent une expertise mais également quand il s'agit de bénévoles comme c'est parfois le cas dans la téléphonie sociale.

\section{Conclusion}

37 Cette relecture orientée sur la mise en jeu du corps dans l'activité des centres d'appel permet de distinguer deux types de communication. La première correspond à l'échange téléphonique guidé avec un client. Le travail relève d'une tâche de nature cognitive qui sollicite essentiellement la parole, l'ouie et la vue; le dispositif entrave toute mobilité corporelle, ne facilitant pas les échanges émotionnels et l'évacuation du stress.

La deuxième relève des "communications ordinaires" qui exigent un corps en mouvement, débranché du dispositif technique. Le re-travail des émotions et des tensions propres aux échanges téléphoniques est alors possible. Celles-ci permettent l'échange de savoir-faire, de pratiques, de valeurs qui donnent sens au travail. Autrement dit; ces espaces où les corps peuvent se mouvoir permettent une activité d'information élargie où les acteurs se sentent autorisés à contribuer au système collectif, comme par exemple, à la mise en cohérence du référentiel d'appels qui organise et prescrit le travail. Cette activité exige de l'autonomie, c'est pourquoi on l'observe essentiellement dans les centres d'appels où les salariés possèdent une certaine expertise. 
et intercalaire social, cet espace entre l'organisation et l'individu où les corps se rencontrent semble la garantie de bonnes conditions de travail dans ces centres d'appel. En effet, cet espace permet à la fois le maintien et l'intégrité physique et mentale des travailleurs par le re-travail des émotions que suscitent les communications téléphoniques, tout en améliorant les pratiques grâce aux échanges de savoir-faire, de modes d'action, de valeurs, et d'agir créatif que ces espaces permettent. Cependant, si les acteurs en sont privés, «c'est le cercle vicieux du sous-développement professionnel: sans moyen collectif de faire face au réel, l'activité individuelle cherche à s'abriter sans succès derrière la tâche prescrite " (Clot, 2008, p154). Autrement dit, sans espace pour un agir créatif collectif où les corps sont désentravés, les innovations organisationnelles seront difficiles et les mauvaises conditions de travail dénoncées dans les centres d'appel ne pourront disparaître.

\section{BIBLIOGRAPHIE}

Bidet, A., (2007a), «La mise en récit du trafic téléphonique. Prendre prise sur un travail « immatériel », Raison présente, n¹62, Nouveaux aspects du travail, p 19-31.

Bidet, A., (2007b), « Le travail entre corps et technique : du labor à l'agir créatif », Communications EHESS, Corps et techniques, $\mathrm{n}^{\circ} 81$, Seuil, p 215-223.

Bouvet, C., Schneider, F., (2007), « Les destins de l'agressivité dans les activités de service client en centre d'appel », Travailler, ${ }^{\circ} 18, \mathrm{p}$ 203-218.

Buscatto, M., (2002), « Les centres d'appels, usines modernes? Les rationalisations paradoxales de la relation téléphonique ", Sociologie du travail, n44, p 99-117.

Cadéac, B., Ferrarini, Y., Oddoux, K., (2004), « L'éducation pour la santé peut aussi passer par le téléphone», La Santé de l'homme, $n^{\circ} 373$, http://www.cfes.sante.fr/SLH/articles/373/01.htm

Chauvière, M., Frotiée, B., (1998), « La Téléphonie sociale. Perspectives sociologiques », Revue Française des Affaires Sociales, $n^{\circ} 2$, La Documentation française, $\mathrm{p}$ 167-180.

Clergeau, C., Pihel, L., Benion, A., (2009), «Les métiers des centres d'appels : des difficultés aux fonctions et choix manégariaux de demain ». Colloque "les perspectives des métiers au service de la GRH ». Essec/IAE/Dauphine. 19 mars 2009.

Clot, Y., (1999), La fonction psychologique du travail. Paris : PUF.

Clot, Y., (2008), Travail et pouvoir d'agir, PUF, coll. Le travail humain, 312p.

Dumez H., (2007), « La créativité de l'agir et l'analyse de l'action située », le Libellio d'AEGIS, vol.3, $\mathrm{n}^{\circ} 4, \mathrm{p}$ 41-45.

Durand, J-P., (2003), La chaine invisible. Travailler aujourd'hui : flux tendu et servitude volontaire. Le Seuil. Paris. 386p.

Guyot, B., (2002), « Mettre en ordre les activités d'information, nouvelle forme de rationalisation organisationnelle », Les Enjeux de l'information et de la communication. http://www.u-grenoble3.fr/ les_enjeux 
Kogan, A-F., (2008), L'ancrage social de la téléassistance pour personnes âgées : des actes de communication à l'information organisationnelle. Les Enjeux de l'information et de la communication. Mise en ligne le 20 mars 2009. http://www.u-grenoble3.fr/les_enjeux

Licoppe, C., (2008), « Aux limites du paradigme de la distribution : l'écoute de détresse et le traitement de la souffrance des suicidaires, du téléphone à l'e-mail », Sociologie du travail, $n^{\circ} 50, p$ 417-433.

Macé, C., (2006), «Les mots de la nuit. Etude sur les appels nocturnes dans un service de téléassistance ». Gérontologie et société, n¹16. Mars, p 233-238.

Michaux, V., Rowe, F., (2004), « Complémentarité entre système d'information informatisé, communauté de pratiques et vigilance dans la haute fiabilité : le cas d'une compagnie d'assistance », SIM, $n^{\circ} 1, \mathrm{p}$ 49-78.

Pichault, F., Zune, M., (2000), « Une figure de la déréglementation du marché du travail : le cas des centres d'appels ", Management et Conjoncture sociale, $\mathrm{n}^{\circ}$ 580. Les Enjeux de l'information et de la communication (2001). http://www.u-grenoble3.fr/les_enjeux.

Sarfati, F., (2008), « L'expérience du stress, son cadre social et ses usages managériaux » in $A u-$ delà du stress au travail, sous la dir. de Buscatto, Loriol, Weller, ERES, coll. Clinique du travail, $\mathrm{p}$ 23-37.

\section{RÉSUMÉS}

Apparu au milieu des années quatre vingt dix, les centres d'appels téléphoniques sont fondés sur une alliance du téléphone et de l'informatique. Ces organisations sont donc exemplaires pour analyser les activités de communication et d'information.

L'approche par l'activité, en redonnant une place à la corporéité permet de saisir d'autres éléments du travail que la seule communication téléphonique. Ainsi, la lecture des travaux sur les centres d'appels montre qu'il existe des espaces où les corps sont désentravés car débranchés du dispositif. Ces espaces permettent, d'une part, le re-travail des chocs émotionnels induits par la relation téléphonique et, d'autre part, la création d'une dynamique collective pour faire évoluer le système d'information au gré du travail. Sans ces espaces pour un agir créatif collectif, les mauvaises conditions de travail dénoncées dans les centres d'appel ne pourront disparaître.

Call centers, that have emerged in the eighties, are built on vocal and data technologies. So, they stand as a proper object for analysis of information and communication activities. Activities approach lets place to body and can get other work practices than call phone only. So, literature about call centers shows places where the body is unshacked because it is unpluged of the technical device. These places let, on the one hand, to do the psychic elaboration to absorb the phone call stress, and, on the other hand, to create a group dynamism to involve the IT device. These places allow groups to exist for creative work and are the conditions to reduce bad working conditions in call centers.

\section{INDEX}

Mots-clés : activité d'information, centre d'appels, corporéité, téléphonie, conditions de travail, organisation du travail

Keywords : information activity, call centers, corporeality, telephony, working conditions, work organization 


\section{AUTEUR}

\section{ANNE-FRANCE KOGAN}

Anne-France Kogan est ingénieure, maître de conférences en Sciences de l'Information et de la Communication au département SSG (sciences sociales et de gestion) de l'Ecole des Mines de Nantes, et membre du LEMNA. Elle enseigne et effectue ses recherches sur le triptyque :

modalités de diffusion des Tic - changements organisationnels - évolution des professions. 